\title{
Simulador de tráfico vehicular mediante el modelo matemático macroscópico LWR
}

\section{Vehicular traffic simulator using the LWR macroscopic mathematical model}

\section{Ángel Gustavo Moyolema Chaglla ${ }^{1 *}$, Francisco Eduardo Toscano Guerrero ${ }^{1}$, Byron Miguel Toalombo Rojas ${ }^{1}$}

1 Universidad Técnica de Ambato, Ecuador

*angelgus@hotmail.es

DOI: https://doi.org/10.26871/killkanatecnica.v5i1.959

\section{Resumen}

Se realiza una simulación de tráfico vehicular mediante un modelo matemático macroscópico para representar el flujo vehicular en un tramo de una avenida de la ciudad de Ambato, Ecuador. En una fase de campo inicial se recolectaron datos de densidades de tráfico, velocidades medias de circulación y flujos de tráfico en cuatro subtramos que comprende el sistema vial, en diferentes días y horarios. Posteriormente se desarrolló un modelo Lighthill-WhithamRichards, resuelto mediante una técnica de solución numérica con determinadas condiciones de frontera y la incorporación de modelos de regresión lineal que expresan la relación entre la 
velocidad y densidad de tráfico. Entre los resultados se destaca que los mayores flujos se presentan a las 18:30 horas, con un pico máximo de 1140 vehículos/h, las densidades de tráfico son similares para los cuatro subtramos, siendo el mayor de 16 autos por subtramo. Existe un subtramo (BA) que presenta la mayor fluctuación de velocidad y densidad de tráfico, siendo el más conflictivo porque tiende a restringir el libre flujo de autos. A partir de los tiempos de semaforización en las entradas y salidas del sistema se efectúan $\mathrm{N}$ simulaciones en un lapso de una hora y al final se contabilizan la cantidad de autos que circulan por la avenida en ese tiempo.

Palabras clave: Densidad de tráfico, flujo de tráfico, modelo LWR, regresión lineal, tiempo, velocidad.

\begin{abstract}
A traffic simulation is carried out using a macroscopic mathematical model to represent the traffic flow in an avenue section in Ambato, Ecuador. In an initial field stage, data on traffic densities, average traffic speeds and traffic flows were registered in four segments of the road system during different days and times. Subsequently, a Lighthill-Whitham-Richards model was developed, which was solved using a numerical solution technique with certain limit conditions and incorporating linear regression models that express the relationship between traffic speed and density. The results show that the highest flows occur at 18:30 hours, with a maximum flow of 1140 vehicles/h. The traffic densities are similar for the four subsections, the highest peak being 16 cars per sub-section. The subsection (BA) has the most significant fluctuation in speed and traffic flow density, and it is the most difficult because it restricts the free traffic flow. $\mathrm{N}$ simulations are carried out during an hour based on the traffic light times at the system's entrances and exits. The number of cars circulating on the avenue is counted at the end.
\end{abstract}

Keywords: traffic density, traffic flow, LWR model, linear regression, time, speed. 


\section{Introducción}

La movilización vehicular en las ciudades afronta algunos problemas como la congestión y la ocurrencia de accidentes de tráfico, los cuales tienden a aumentar progresivamente con el incremento del parque automotor, sumado a otros factores propios de determinados lugares, como la existencia de una topografía montañosa. La modelación del tráfico vehicular se aborda desde algunos enfoques particulares. En este sentido, los simuladores de tráfico pueden ser microscópicos o macroscópicos, según el nivel de detalle requerido.

El enfoque macroscópico se caracteriza por variables de tipo colectivo, por lo que los vehículos son indistinguibles, se proyectan solamente cantidades promediadas; por esta razón se requiere que se tenga bajo consideración un gran número de vehículos en circulación.

Entre las principales ventajas de los modelos macroscópicos se destaca que los resultados son cualitativamente cercanos al comportamiento real, existe una buena capacidad de análisis de las ecuaciones establecidas y son computacionalmente más eficientes que los microscópicos. El número de variables involucradas desempeña un rol fundamental en el nivel de detalle de la descripción del fenómeno [1].

Las simulaciones de tráfico en los sistemas viales suelen tener la capacidad de representar y proporcionar información respecto al ritmo de arribos de vehículos, tiempos de recorrido y de espera, velocidades, predicción de congestionamientos, monitoreo del tráfico, evaluación de medidas para controlar el exceso de velocidad y evaluación del funcionamiento de semáforos en intersecciones [2] [3].

El presente trabajo tiene por objeto proporcionar información técnica sobre el comportamiento del tráfico vehicular en un sector urbano que alberga a un centro comercial, a través de una simulación, basándose en estudios de modelado macroscópico y haciendo uso de la información obtenida mediante una observación directa en el tramo vial de interés. De esta manera, por intermedio de la manipulación de las variables inherentes al tráfico vehicular (velocidad y densidad de tráfico) y los ciclos de funcionamiento de los semáforos, se plantea la modelación y simulación de los escenarios que describen la variable dependiente flujo vehicular, lo que sirve de referente para la adopción de acciones por parte de los organismos de tránsito municipales para el mejoramiento del flujo vehicular. Desde ese punto de vista, la investigación tiene una relevancia social en pro de contribuir para una eficiente gestión de la movilidad urbana.

Previo a la simulación del tráfico vehicular se requiere la creación del modelo matemático, de manera que la simulación se orienta básicamente en hacer predicciones del comportamiento real del fenómeno. Entre los aspectos inherentes al proceso de modelación y simulación constan la predeterminación de la estructura del modelo, la programación, la validación del modelo, el análisis y la crítica de los resultados.

\section{Marco Teórico}

\section{A. Flujo de tráfico de vehículos}

La dinámica del flujo de tráfico vehicular incluye rangos de escala de tiempo, expresado en segundos, minutos o pocas horas. El avance de los vehículos, definido como el tiempo entre dos vehículos sucesivos cuando pasan por el mismo punto en el mismo carril, es una medida fundamental tanto en la teoría del flujo de tráfico como en las aplicaciones de transporte. El avance caracteriza la forma como se distribuyen los vehículos en una carretera y está relacionado con la tasa de flujo de tráfico. Por otro lado, si el tráfico 
no fluye libremente, el avance del vehículo también refleja cómo los conductores siguen a los vehículos que van delante. Por lo tanto, los datos de avance de los vehículos a menudo describen simultáneamente parámetros macroscópicos de flujo de tráfico y el comportamiento microscópico de seguimiento de vehículos de los conductores [4].

Las magnitudes que generalmente son consideradas en la modelación y simulación del tráfico vehicular son las que se describen a continuación:

\section{B. Flujo de tráfico}

El flujo de tráfico q se define como el número de vehículos $\Delta N$ que atraviesan una sección transversal en una ubicación $x$, dentro de un intervalo de tiempo $\Delta t$. Las unidades de medición de esta magnitud, generalmente son en vehículos por hora (veh/h) o en vehículos por minuto (veh/ $\min )[5]$.

$$
q(x, t)=\frac{\Delta N}{\Delta t}
$$

\section{Densidad de tráfico}

La densidad de tráfico $\rho(x, t)$ es una magnitud que expresa la relación del flujo de tráfico $\mathrm{q}(\mathrm{x}, \mathrm{t})$ respecto a la velocidad lineal media de los vehículos $\mathrm{v}(x, t)$. Se puede obtener una mejor estimación de la densidad de tráfico, a partir de la definición "vehículos por distancia", que se puede expresar en términos de cantidades microscópicas, como la inversa de la media espacial de las distancias de avance. Las unidades en las que se suele medir la densidad de tráfico son en vehículos por kilómetro (veh/km) o vehículos por metro (veh/m) (Treiber $\&$ Kesting, 2013, pp. 17-18). La densidad se denota por medio de la siguiente expresión:

$$
\rho(x, t)=\frac{q(x, t)}{\mathrm{v}(x, t)}
$$

\section{Velocidad media}

La velocidad lineal media (media instantánea) $\mathrm{v}(\mathrm{t})$ es la media aritmética de la velocidad de todos los vehículos dentro de un segmento de carretera dado en un tiempo t. Siendo n(t) el número de vehículos en el tramo de carretera referenciado, la velocidad media se expresa de la siguiente manera:

$$
\mathrm{v}(t)=\frac{1}{n(t)} \sum_{\mathrm{i}=1}^{n(t)} \mathrm{v}_{\mathrm{i}}(t)
$$

\section{E. Relación de la densidad, velocidad y flujo}

La relación de las magnitudes densidad de tráfico, velocidad y flujo, se puede expresar a través de la ecuación fundamental del flujo vehicular (ecuación 4) y de la ecuación diferencial parcial que describe la conservación de los vehículos (ecuación 5). Paralelamente, es posible incorporar las magnitudes fundamentales espacio $\mathrm{x} y$ tiempo $t$, con la finalidad de analizar el comportamiento vehicular en un determinado tramo y en un intervalo de tiempo definido.

De esta manera, la densidad de tráfico es una función de la forma $\rho(x, t)$ y el flujo otra de la forma $q(x, t)$, para un tiempo $t \in\left[0, \mathrm{t}_{\mathrm{m}}\right]$ y una posición $x \in\left[x_{0}, x_{n}\right][6]$. A partir de las funciones indicadas es posible contar con una expresión que relacione las cinco magnitudes mediante un modelo matemático de tráfico, como el Lighthi1l-Whitham-Richards (LWR) [7].

$$
\begin{gathered}
q=\rho \cdot \mathrm{v} \\
\frac{\partial \rho}{\partial t}+\frac{\partial q}{\partial x}=0
\end{gathered}
$$

\section{F. Modelo de tráfico de Lighthill-Whitham-Ri- chards}

El modelo Lighthill-Whitham-Richards (LWR) corresponde a un modelo de tipo macroscópico 
de tráfico vehicular unidimensional, en el que se considera que ningún vehículo ingresa o sale de la carretera, lo que permite pensar que se conserva el número de vehículos. La ecuación del modelo LWR tiene como punto de partida la conservación de la masa en forma diferencial [7].

Sustituyendo (4) en (5) se tiene la ecuación diferencial parcial del modelo LWR:

$$
\frac{\partial \rho}{\partial t}+\frac{\partial(\rho v)}{\partial x}=0
$$

\section{G Método de características o técnica analítica de resolución}

Este método de resolución de una ecuación diferencial parcial consiste en encontrar una solución al problema, a partir de las condiciones iniciales y de frontera, así como con la utilización de funciones existentes que relacionan a las variables involucradas.

Particularmente es de interés la utilización de una función que exprese la velocidad a partir de la densidad de tráfico, es decir v( $\rho)$. Para el efecto se puede partir de ciertos modelos teóricos existentes basados en funciones como la de Greenshields de la ecuación (7), Greenberg, Underwood y Pipes-Murigel, que son de tipo lineal, logarítmica, exponencial o polinomial, respectivamente [8].

$$
\mathrm{v}=\mathrm{v}_{\text {máx }}\left(1-\frac{\rho}{\rho_{\text {máx }}}\right)
$$

La ecuación de Greenshields (7) es la más ampliamente utilizada y la que entraña una menor complejidad. Otra alternativa viable al uso de los modelos teóricos, es la obtención de una función a partir de la recolección de datos en campo, con la finalidad de hallar regresiones lineales o polinomiales que expresen la velocidad en función de la densidad de tráfico $v(\rho)$. El caso más simple corresponde a los modelos de regresión lineal, que se expresa de la siguiente manera:

$$
\mathrm{v}=\beta_{0}+\beta_{1} \cdot \rho
$$

Donde:

$\beta_{0}$ intercepto de la función lineal obtenida mediante regresión.

$\beta_{1}$ pendiente de la función lineal obtenida mediante regresión.

Derivando la expresión (8) con respecto a $d \rho$ para hallar los puntos críticos se tiene:

$$
\begin{gathered}
\frac{\mathrm{dq}}{\mathrm{d} \rho}=\beta_{0}+2 \beta_{1} \cdot \rho \\
\frac{\mathrm{dq}}{\mathrm{d} \rho}=0 \rightarrow \rho=-\frac{\beta_{0}}{2 \beta_{1}} \quad \text { punto crítico }
\end{gathered}
$$

Al aplicar la segunda derivada y dado que la pendiente $\beta 1$ es negativa porque la relación velocidad es inversamente proporcional a la densidad de tráfico, se tiene:

$$
\begin{gathered}
\frac{\mathrm{d}^{2} \mathrm{q}}{\mathrm{d} \rho^{2}}=2 \beta_{1}<0 \\
\rho=-\frac{\beta_{0}}{2 \beta_{1}} \text { punto crítico máximo }
\end{gathered}
$$

El flujo máximo queda expresado de la siguiente manera:

$$
\begin{gathered}
q=\beta_{0} \cdot \rho+\beta_{1} \cdot \rho^{2} \\
q_{\text {máx }}=-\frac{\beta_{0}^{2}}{4 \beta_{1}}
\end{gathered}
$$

La velocidad máxima cuando el flujo de tráfico es máximo es:

$$
\mathrm{v}_{\text {máx }}=\frac{\beta_{0}}{2}
$$




\section{H Técnica de solución numérica}

La resolución analítica halla soluciones para los valores máximos y mínimos de las magnitudes densidad de tráfico, velocidad y flujo, poniendo atención en los puntos máximos y mínimos. Sin embargo, es necesario definir el comportamiento a partir del tiempo t y el espacio $x$, razón por la cual es necesario efectuar un análisis de diferencias finitas, que permita determinar las densidades de tráfico a partir de las densidades anteriores en una ubicación específica de un tramo y en un instante de tiempo concreto. Para el efecto se hace una discretización del dominio en subintervalo de tiempo y espacio.

A partir de la ecuación (6) del modelo LWR se tiene:

$$
\begin{gathered}
\frac{\partial \rho}{\partial t}+\rho \frac{\partial \mathrm{v}}{\partial x}+\mathrm{v} \frac{\partial \rho}{\partial x}=0 \\
\frac{\partial \rho}{\partial t} \approx \frac{\rho(x, t+\mathrm{d} t)-\rho(x, t)}{\mathrm{d} t}=\frac{\rho(\mathrm{i}, \mathrm{j}+1)-\rho(\mathrm{i}, \mathrm{j})}{\mathrm{d} t} \\
\frac{\partial \mathrm{v}}{\partial x} \approx \frac{\mathrm{v}(x, t)-\mathrm{v}(x-\mathrm{d} x, t)}{\mathrm{d} x}=\frac{\mathrm{v}(\mathrm{i}, \mathrm{j})-\mathrm{v}(\mathrm{i}-1, \mathrm{j})}{\mathrm{d} x} \\
\frac{\partial \rho}{\partial x} \approx \frac{\rho(x, t)-\rho(x-\mathrm{d} x, t)}{\mathrm{d} x}=\frac{\rho(\mathrm{i}, \mathrm{j})-\rho(\mathrm{i}-1, \mathrm{j})}{\mathrm{d} x}
\end{gathered}
$$

Sustituyendo en (17):

$$
\begin{aligned}
\frac{\rho(i, j+1)-\rho(i, j)}{d t} & +\rho(i, j) \frac{v(i, j)-v(i-1, j)}{d x} \\
& +v(i, j) \frac{\rho(i, j)-\rho(i-1, j)}{d x}=0
\end{aligned}
$$

Multiplicando a toda la expresión por $d t$, se tiene el Esquema de diferencias finitas para el modelo LWR:

$$
\begin{array}{r}
\rho(i, j+1)=\rho(i, j)-\frac{d t}{d x}[\rho(i, j)[v(i, j)-v(i-1, j)] \\
+v(i, j)[\rho(i, j)-\rho(i-1, j)]]
\end{array}
$$

De acuerdo a Greenshields:

$$
v(i, j)=v_{\text {máx }}\left[1-\frac{\rho(i, j)}{\rho_{\text {máx }}}\right]
$$

\section{Materiales y métodos}

\section{A. Metodología}

La presente investigación es de tipo explicativa, puesto que a partir de la descripción del fenómeno del tráfico vehicular en la avenida Manuelita Sáenz comprendido entre las calles Pío Baroja y Antonio Clavijo de la ciudad de Ambato y la identificación de las variables involucradas, se plantea el establecimiento de las relaciones causales en los eventos. De manera que se pueda ejecutar una simulación de los distintos escenarios del flujo del tráfico vehicular.

\section{B. Ubicación del sitio de estudio}

La zona de estudio, que se muestra en la Figura 1 , consta de tres intersecciones, de entre las cuales dos son semaforizadas (A y C). No obstante, dichas intersecciones son las causantes de la presencia de eventuales cuellos de botellas, al mismo tiempo que definen la capacidad vehicular, el flujo vehicular y la densidad de tráfico que circula por el sector. En medio de las dos intersecciones semaforizadas, existe una intersección no semaforizada (B), a partir de la observación de campo, se evidenció que esta última tiene un flujo de tráfico pequeño.

Otro aspecto a tener en cuenta es que el ingreso de los vehículos hacia las instalaciones del centro comercial es por la avenida Manuelita Sáenz, mientras que la salida de los vehículos del interior del centro comercial es por la calle Pío Baroja, que tiene un solo sentido hasta la desembocadura en la avenida Manuelita Sáenz.

La resolución se desarrolla mediante un proceso iterativo. 


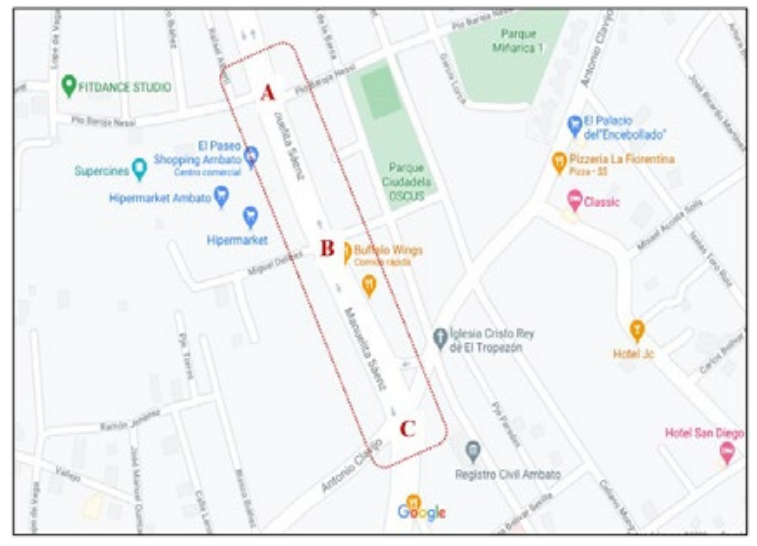

FIG. 1. Localización del tramo vehicular del estudio.

\section{Tipo de investigación}

La presente investigación es de tipo explicativa, puesto que a partir de la descripción del fenómeno del tráfico vehicular en la avenida Manuelita Sáenz comprendido entre las intersecciones A y C y la identificación de las variables involucradas, se plantea el establecimiento de las relaciones causales en los eventos. Se utilizan los datos obtenidos en el estudio de campo para cada uno de los subtramos AB, BC, CB y BA. De esta manera se ejecuta una simulación de los distintos escenarios del flujo del tráfico vehicular.

\section{Población y muestra}

La población está conformada por el número de vehículos que circulan en el tramo de la avenida Manuelita Sáenz comprendido entre las intersecciones A y B. En vista de que la cantidad de la población depende del intervalo de tiempo considerado, es necesario establecer un lapso de interés.

En este sentido, debido a que se tiene por finalidad la simulación de diferentes escenarios de tráfico vehicular en el tramo de estudio, se optó por efectuar un muestreo no probabilístico por conveniencia mediante la realización de observaciones en distintos días y a diferentes horarios, teniendo como criterio las horas de mayor tránsito vehicular. La observación consistió en el conteo de la cantidad de vehículos que circularon por cada uno de los tramos.

A partir de las mediciones realizadas se dispuso de datos para el modelamiento del tráfico vehicular, los cuales permitieron la estimación del comportamiento en los diferentes escenarios analizados. A continuación, se presenta el detalle de los días y horas en los que se efectúa la medición del tráfico vehicular.

TABLA I:

MUESTRA PARA LA OBTENCIÓN DE DATOS DE TRÁFICO VEHICULAR

\begin{tabular}{lccc}
\hline \multicolumn{1}{c}{ Día } & Hora & No. mediciones & $\begin{array}{c}\text { Tiempo de observación } \\
\text { por medición }\end{array}$ \\
\hline Lunes a viernes & $08 \mathrm{~h} 30$ & 5 & $1 \mathrm{~h}$ \\
\hline Lunes a viernes & $11 \mathrm{~h} 00$ & 5 & $1 \mathrm{~h}$ \\
\hline Lunes a viernes & $13 \mathrm{~h} 30$ & 5 & $1 \mathrm{~h}$ \\
Lunes a viernes & $18 \mathrm{~h} 30$ & 5 & $1 \mathrm{~h}$ \\
Lunes a viernes & $20 \mathrm{~h} 00$ & 5 & $1 \mathrm{~h}$ \\
Sábados y domingos & $10 \mathrm{~h} 00$ & 2 & $1 \mathrm{~h}$ \\
Sábados y domingos & $13 \mathrm{~h} 00$ & 2 & $1 \mathrm{~h}$ \\
Sábados y domingos & $17 \mathrm{~h} 00$ & 2 & $1 \mathrm{~h}$ \\
Sábados y domingos & $20 \mathrm{~h} 00$ & 2 & $1 \mathrm{~h}$ \\
\multicolumn{1}{c}{ Total } & - & 33 & -
\end{tabular}


Como criterio de inclusión se contabilizaron los autos livianos y pesados que circularon por cada uno de los tramos durante los periodos de observación, pero se excluyeron a las motocicletas y bicicletas, debido a que el flujo fue despreciable y debido a que no representan medios de transporte que ocasionen congestión vehicular en el sector.

\section{E. Recolección de datos}

La técnica utilizada para la recolección de los datos fue la observación directa y el registro correspondiente de los datos obtenidos, que correspondieron a la cantidad de vehículos que circularon por cada tramo de estudio durante el lapso de medición. Para llevar a cabo el proceso de recopilación de datos, en primera instancia se identificaron las tres intersecciones. A continuación, se presenta la información de cada uno de los subtramos correspondientes:

TABLA II:

SUBTRAMOS DELIMITADOS POR LAS INTERSECCIONES

\begin{tabular}{cccc}
\hline Subtramo & Sentido & Distancia & Semaforización \\
\hline A-B & Norte-Sur & $155 \mathrm{~m}$ & 8 semáforos ve- \\
B-A & Sur- Norte & $140 \mathrm{~m}$ & $\begin{array}{c}\text { hiculares en la } \\
\text { intersección A }\end{array}$ \\
& & & 9 semáforos ve- \\
B-C & Norte-Sur & $180 \mathrm{~m}$ & hiculares en la \\
C-B & Sur- Norte & $175 \mathrm{~m}$ & intersección C
\end{tabular}

Mediante la relación de la cantidad de vehículos que circularon por cada intersección con respecto al tiempo de duración de cada lapso de observación se obtuvo los flujos vehiculares, expresado en vehículos por hora (veh/h). La notación empleada para cada flujo vehicular y el sentido de circulación vehicular se ilustra en los siguientes gráficos:

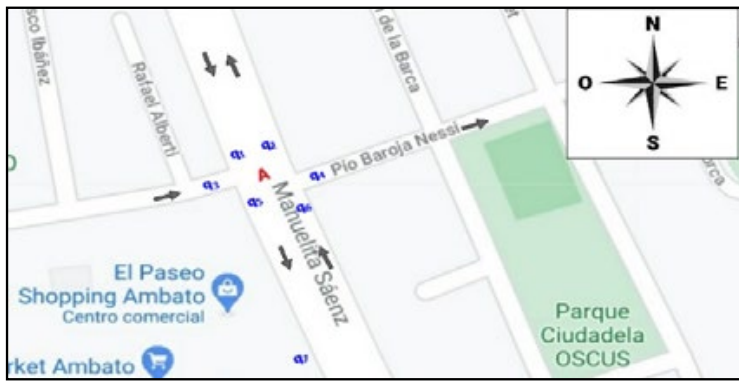

FIG. 2. Identificación de los flujos de tráfico y el sentido, intersección A.

El flujo q7 corresponden al ingreso al centro comercial ubicado en el tramo de estudio.

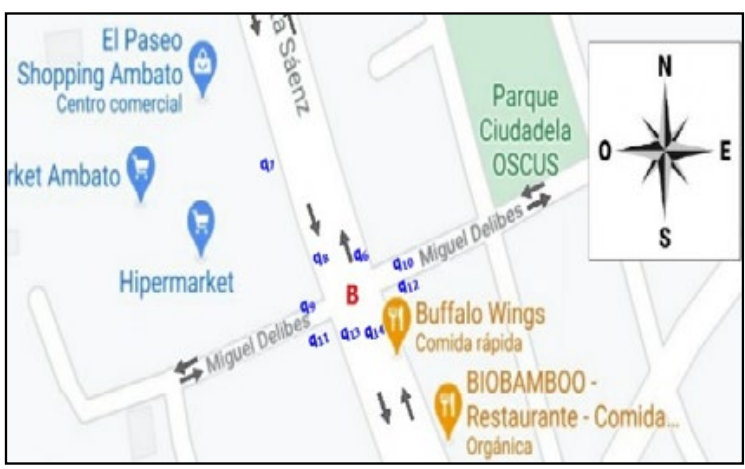

FIG. 3. Identificación de los flujos de tráfico y el sentido, intersección B.

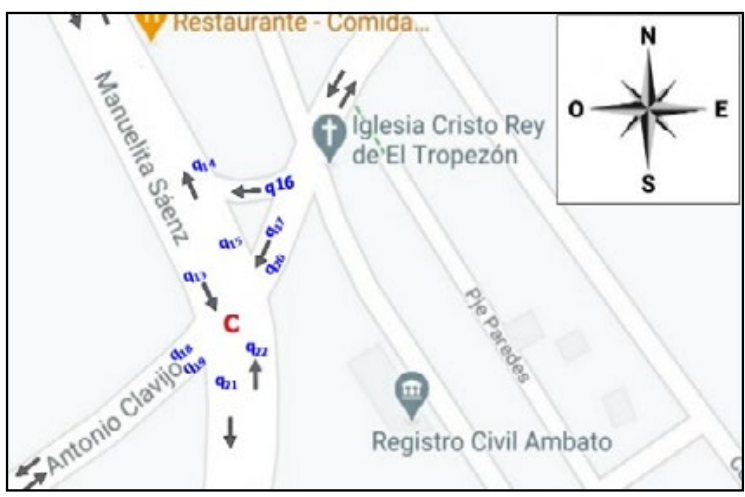

FIG. 4. Identificación de los flujos de tráfico y el sentido, intersección C.

\section{F. Análisis de datos}

El procesamiento de la información y análisis de datos se llevó a cabo con el apoyo de un software de cómputo numérico, conforme se describe a continuación:

Una vez recopilados los flujos de tráfico a través de las mediciones efectuadas en los cuatro subtramos 
del sistema estudiado, se establecieron los valores mínimos, máximos y promedios para los diferentes días de la semana y en los horarios observados. Los valores encontrados sirvieron para establecer los rangos de fluctuación de cada uno de los flujos de tráfico, teniendo en cuenta los valores máximos y mínimos encontrados. Adicionalmente se elaboraron diagramas de caja para identificar los cuartiles de los flujos de tráfico de cada subtramo.

Se determinaron las probabilidades de ocurrencia de los 22 flujos de tráfico en cada uno de los accesos, salidas e interior del sistema. Para la toma de los datos de densidades de tráfico se efectuaron 40 recorridos en vehículo a través de cada uno de los subtramos de la avenida Manuelita Sáenz tanto en el sentido norte-sur, como en el sur-norte. Se registraron los tiempos de cada recorrido, al mismo tiempo que el número de autos que circularon por cada subtramo.

A partir de los datos de tiempo y conociendo la longitud de cada subtramo se determinaron las velocidades medias a la que circulan los vehículos, las cuales se esperaba que no excedan de $50 \mathrm{~km} / \mathrm{h}$, que es la velocidad máxima en el sector urbano según la institución encargada de la regulación de tránsito en Ecuador. Se obtuvieron diagramas de caja para representar los valores mínimos, máximos y los cuartiles de las densidades de tráfico y de las velocidades.

Los datos de densidad de tráfico y velocidad fueron utilizados para establecer una función lineal, que expresa la relación de la velocidad en función de la densidad de tráfico, la cual fue obtenida mediante una regresión lineal. La simulación de tráfico se realizó mediante la aplicación del modelo LWR para generar densidades de tráfico, velocidades y flujos en cada uno de los subtramos del sistema.

Los flujos de tráfico se simularon mediante la aplicación de la probabilidad de ocurrencia de cada uno a partir de los datos recopilados. Los flujos de entrada se generaron de forma aleatoria, al igual que los flujos interiores que se obtuvieron de la simulación del modelo macroscópico LWR. De esta manera se calcularon los flujos de salida del sistema, considerando las mismas probabilidades de ocurrencia.

\section{G. Variables respuesta}

Las variables de respuesta del sistema se detallan en la Tabla 3:

TABLA III:

SUBTRAMOS DELIMITADOS POR LAS INTERSECCIONES

\begin{tabular}{|c|c|c|}
\hline Aspecto & $\begin{array}{c}\text { Variables de } \\
\text { entrada }\end{array}$ & $\begin{array}{c}\text { Variables de } \\
\text { respuesta }\end{array}$ \\
\hline $\begin{array}{l}\text { Simulación de } \\
\text { tráfico a través } \\
\text { del modelo } \\
\text { macroscópico } \\
\text { LWR. }\end{array}$ & $\begin{array}{c}\text { Intervalos de } \\
\text { espacio dentro } \\
\text { de los tramos y } \\
\text { tiempo. } x, t\end{array}$ & $\begin{array}{c}\text { Densidad de } \\
\text { tráfico, velo- } \\
\text { cidad y flujo } \\
\text { de tráfico. } \rho \text {, } \\
\text { v, q. }\end{array}$ \\
\hline $\begin{array}{l}\text { Función lineal } \\
\text { para definir la } \\
\text { densidad de } \\
\text { tráfico vs. la } \\
\text { velocidad. }\end{array}$ & $\begin{array}{l}\text { Densidad de } \\
\text { tráfico. } \rho\end{array}$ & Velocidad, v. \\
\hline $\begin{array}{l}\text { Flujo de tráfico } \\
\text { vehicular a tra- } \\
\text { vés de probabili- } \\
\text { dad de ocurren- } \\
\text { cia del evento. }\end{array}$ & $\begin{array}{c}\text { Flujos de trafico } \\
\text { de entrada al } \\
\text { sistema. } \mathrm{q}_{1}, \mathrm{q}_{3}, \\
\mathrm{q}_{10}, \mathrm{q}_{11}, \mathrm{q}_{16}, \mathrm{q}_{17}, \\
\mathrm{q}_{19}, \mathrm{q}_{22} \text {. } \\
\text { Flujos interiores } \\
\mathrm{q}_{5}, \mathrm{q}_{13}, \mathrm{q}_{14} \text { y } \mathrm{q}_{6} .\end{array}$ & $\begin{array}{l}\text { Flujos de tra- } \\
\text { fico de salida } \\
\text { al sistema. } \\
\mathrm{q}_{2}, \mathrm{q}_{4}, \mathrm{q}_{7}, \mathrm{q}_{8}, \\
\mathrm{q}_{9}, \mathrm{q}_{12}, \mathrm{q}_{15}, \\
\mathrm{q}_{18}, \mathrm{q}_{20}, \mathrm{q}_{21} .\end{array}$ \\
\hline
\end{tabular}

\section{Resultados y discusión}
A. Simulación del modelo LWR con una función lineal obtenida mediante regresión
La simulación del tráfico vehicular permitió la de- terminación de las posiciones y tiempos dentro de cada uno de los subtramos, para lo cual se aplicó la ecuación del modelo LWR de la ecuación (18). En 
cada simulación se generó una matriz de datos de espacio $\mathrm{x} y$ tiempo $\mathrm{t}$ a partir de la que se obtienen los flujos de tráfico, las velocidades y los flujos.

Con la finalidad de tomar en cuenta los tiempos de semaforización vigentes en el período de las observaciones, se procedió a generar un número de eventos $\mathrm{N}$ producidos en un lapso de tiempo de una hora reloj.

La cantidad de eventos se obtuvo de la relación del tiemplo total de 1 hora sobre la suma de los tiempos de duración de las luces amarilla, verde y rojo del semáforo. Adicionalmente se generaron valores aleatorios de densidad y velocidad iniciales, como punto de partida de la simulación acumulativa de la solución numérica del modelo LWR.

La solución del problema requirió la consideración de la función de la velocidad a partir de la densidad de tráfico, pero ahora mediante el establecimiento de funciones lineales obtenidas de la regresión de los datos recopilados durante el estudio de campo, lo que corresponde al modelo real. Los resultados obtenidos de la simulación se resumen a continuación:

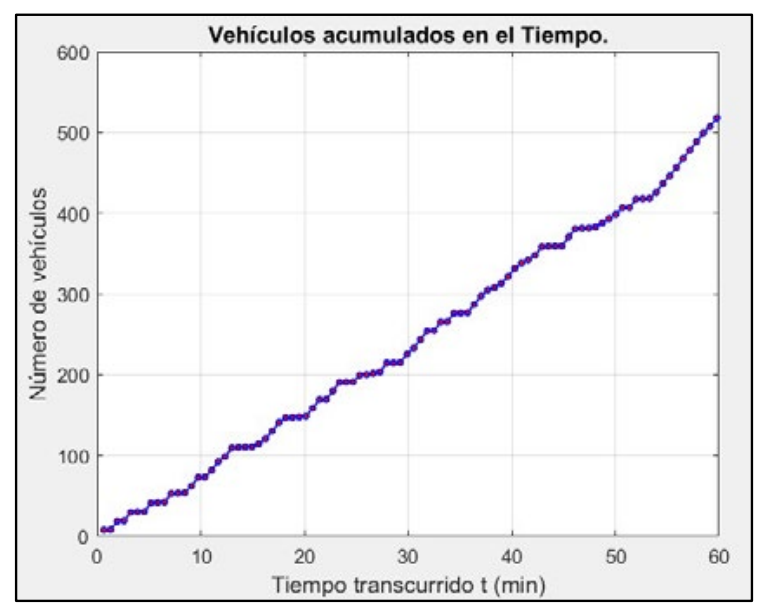

FIG. 5. Simulación del flujo q5 de vehículos en una hora, subtramo $\mathrm{AB}$.

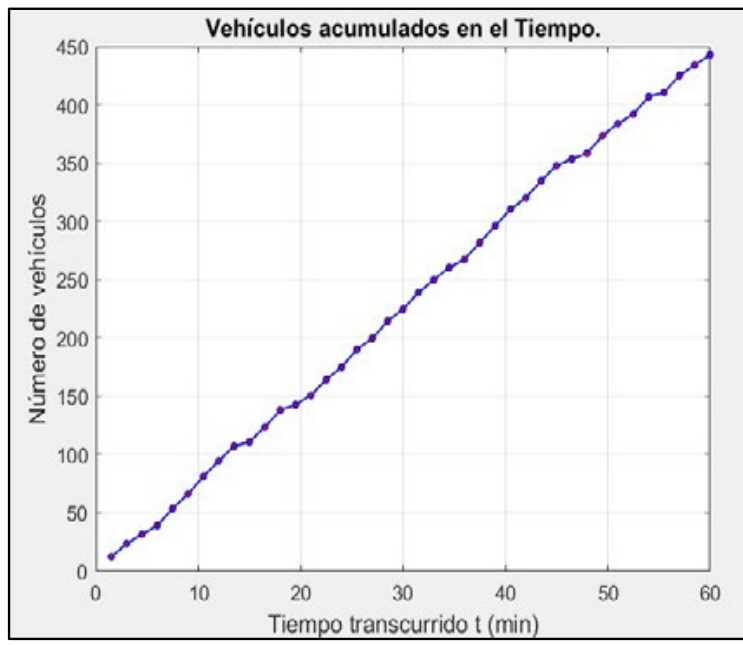

FIG. 6. Simulación del flujo q13 de vehículos en una hora, subtramo BC.

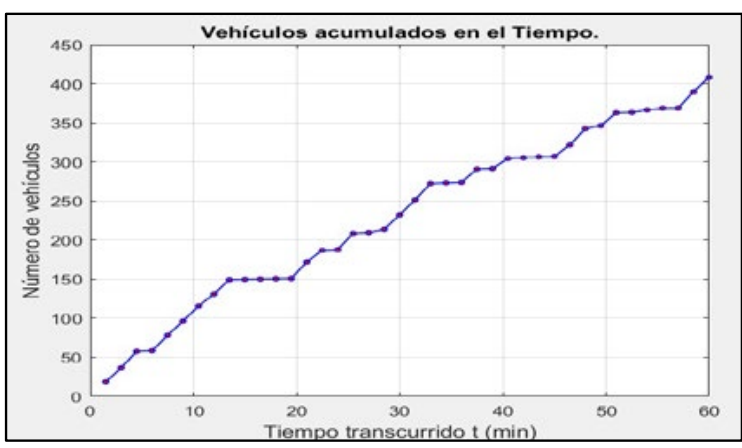

FIG. 7. Simulación del flujo q14 de vehículos en una hora, subtramo CB.

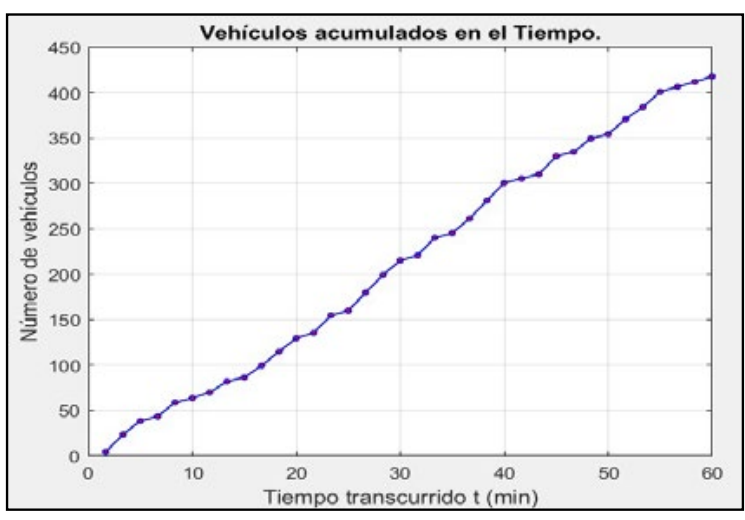

FIG. 8. Simulación del flujo q6 de vehículos en una hora, subtramo BA.

\section{B. Simulación del flujo de tráfico}

La simulación del flujo de tráfico se realizó tomando como referente las probabilidades de ocurrencia de cada una de las salidas en función de 
los flujos de entrada presentados. En este sentido, se crearon en las entradas flujos de tráfico aleatorios en el rango de valores establecidos por el estudio de campo realizado. La codificación se llevó a cabo tomando en cuenta, en primer lugar, la necesidad de obtener las probabilidades de ocurrencia de los flujos de salida en función de los flujos de entrada observados. En la Figura 9 se presenta una captura de pantalla de la codificación utilizada en el software MATLAB:

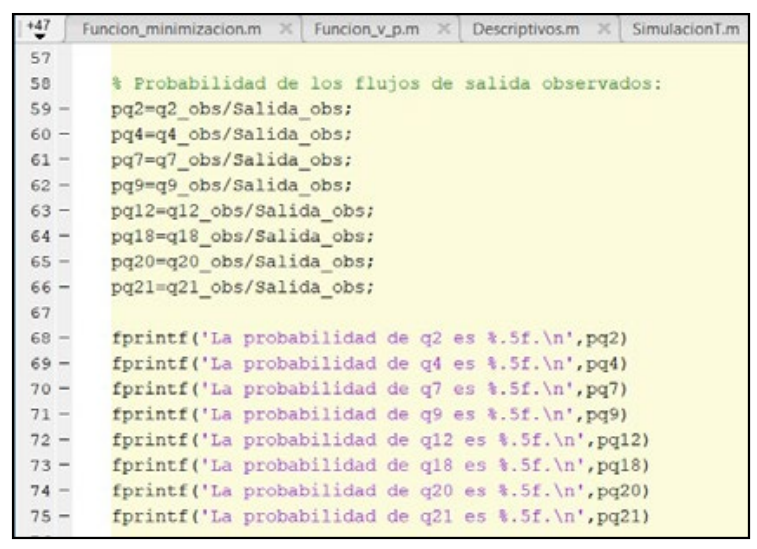

FIG. 9. Simulación del flujo q6 de vehículos en una hora, subtramo BA.

Una vez que se cuentan con las probabilidades, se procedió a configurar la generación de datos aleatorios en los rangos establecidos por el estudio realizado, conforme se muestra en la Figura 10:

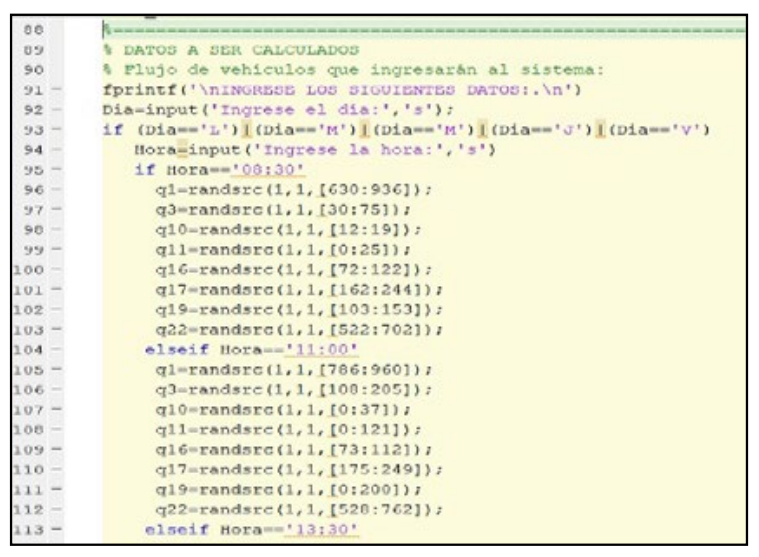

FIG. 10. Codificación para la generación de datos aleatorios del flujo de vehículos que ingresan al sistema.
A continuación, se calcularon los flujos predichos para el sistema, mediante utilización de las probabilidades y los datos aleatorios generados para los flujos de entrada. En el caso de los flujos obtenidos del modelo LWR, q5, q6, q13 y q14, éstos datos se incorporan directamente al script de los flujos de tráfico, mediante lectura de los archivos ".mat" generados del script de simulación de tráfico con el modelo LWR. En la Figura 11 se muestra la codificación correspondiente:

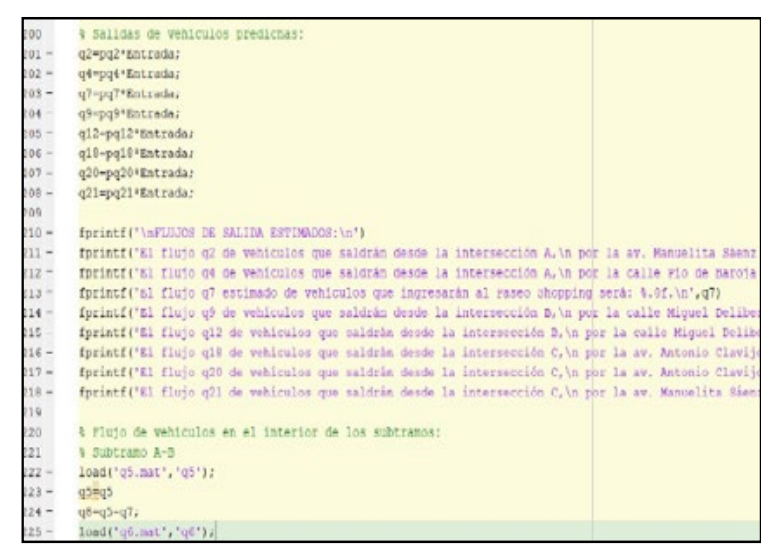

FIG. 11. Programación de los flujos de vehículos que salen del sistema.

Por último, se llevó a cabo una simulación con la codificación establecido y se despliegan los flujos de salida del sistema, como se muestra en la Figura 12. Se efectuó una simulación de las densidades de tráfico en el interior del sistema, considerando la posición y el intervalo de tiempo de los vehículos en cada subtramo. Para el efecto se utilizó el modelo macroscópico de tráfico vehicular LWR que trabaja con las densidades y velocidades. Para hallar los flujos fue necesario incorporar la ecuación fundamental del tráfico que relaciona las tres magnitudes: densidad, velocidad y flujo.

Se efectuaron $N$ simulaciones en un lapso de una hora, tomando en cuenta los tiempos de semaforización, teniendo presente que los autos circulan cuando la luz se encuentra en verde. Cada 
simulación representa un evento y al final se contabilizaron los autos que circularían durante los 60 minutos, lo cual se expresa como el flujo de autos que pasan por el interior del sistema.

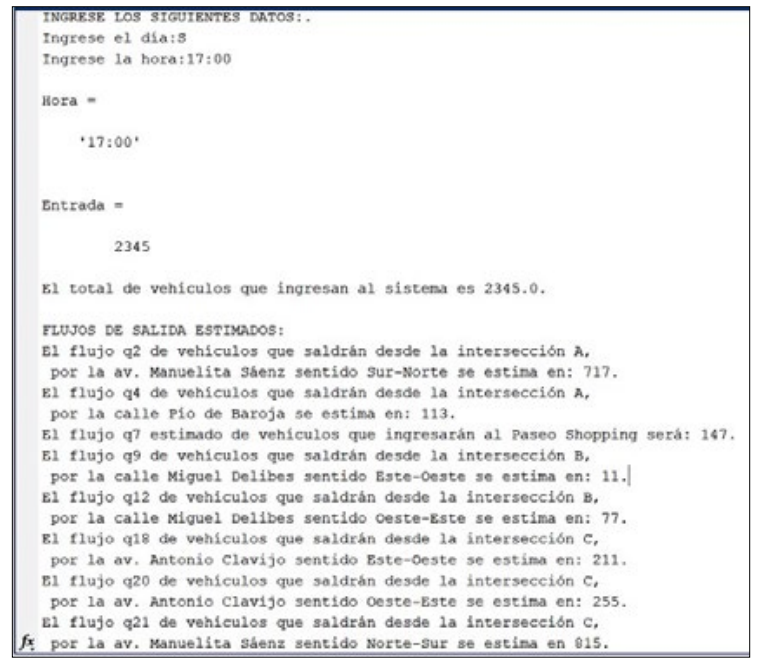

FIG. 11. Programación de los flujos de vehículos que salen del sistema.

\section{Conclusiones}

Las densidades de tráfico en cada uno de los subtramos se obtuvieron mediante observación directa en el lugar de los hechos, así como las velocidades medias de recorrido realizadas por los autos. En términos generales las densidades de tráfico fueron similares para los cuatro subtramos, aunque se destacan los casos de los subtramos BC y BA, con una mayor densidad. El pico máximo alcanzado fue de 16 autos por subtramo.

Se identificó que la hora en la que se presentan los mayores flujos corresponde a las 18:30 en todo el tramo de estudio. El flujo de tráfico más elevado de los registrados fue q1 con 1140 vehículos/h, que corresponde a los vehículos que ingresan desde la avenida Manuelita Sáenz, sentido norte-sur hacia la intersección A.

Las velocidades de recorrido de los vehículos son más elevadas en el subtramo $\mathrm{CB}$, cuyos picos más altos alcanzan el límite de velocidad máximo moderado de $60 \mathrm{~km} / \mathrm{h}$, es decir que exceden los $50 \mathrm{~km} / \mathrm{h}$ que es lo recomendado en el perímetro urbano. Sin embargo, la fluctuación de los datos es pronunciada, pudiendo ser inferior a $20 \mathrm{~km} / \mathrm{h}$. Mediante la regresión lineal se estableció que la velocidad se puede expresar como una función de la densidad de tráfico, con un error mínimo para los cuatro modelos encontrados. El tramo que presentó la mayor fluctuación de velocidad y densidad de tráfico es el BA, lo que denota que es el más conflictivo, dado que restringe el libre flujo de autos de forma más recurrente.

Con la finalidad de particularizar el comportamiento de cada uno de los vehículos, se recomienda la ejecución de un estudio con un modelo microscópico, que sirva de complemento al presente estudio y que permita contrastar los resultados alcanzados.

\section{Agradecimientos}

Expresamos un cordial agradecimiento a la Universidad Católica de Cuenca, por brindarnos su apoyo para la publicación de esta investigación.

\section{Referencias}

[1] A. M. Ramírez-Rabelo, «Modelación Matemática del Tráfico Vehicular con el Modelo "Car-Following"», pp. 2-18, 2019.

[2] P. J. Torres-Vega, «Simulación del tráfico en una vía expresa y análisis estadístico de los resultados», Ingeniería Industrial, n.o 30, pp. 45-79, 2012.

[3] H. Cao y J. Luo, «Research on VISSIM-MATLAB Integrated Traffic Simulation Platform Based on COM Interface Te- 
chnology», en 2019 IEEE 3rd Information Technology, Networking, Electronic and Automation Control Conference (ITNEC), Nangjing, China, mar. 2019, pp. 305-309. doi: 978-1-5386-6243-4.

[4] L. Li y X. Chen, «Vehicle headway modeling and its inferences in macroscopic/microscopic traffic flow theory: A survey», Transportation Research, pp. 170-188, 2017, doi: http://dx.doi.org/10.1016/j. trc.2017.01.007.

[5] M. Treiber y A. Kesting, Traffic flow dynamics: data, models and simulation. Berlin, Germany: Springer, 2013.

[6] P.-E. Mazaré, A. H. Dehwah, C. G. Claudel, y A. M. Bayen, «Analytical and grid-free solutions to the Lighthill-Whitham-Richards traffic flow model», Transportation Research Part B: Methodological, vol. 45, n.o 10, pp. 1727-1748, dic. 2011, doi: 10.1016/j.trb.2011.07.004.

[7] Y. M. Vasquéz y Dr. J. J. Laguardia, «Estudio del Flujo Vehicular Mediante un Modelo de Lighthill-Whitham-Richards», $K E G$, vol. 3, n.o 1, p. 449, 2018, doi: 10.18502/keg.v3i1.1449.

[8] H. Hernández-Vega, «Modelo de Greenshields Un estudio de la capacidad de tráfico», Boletín Técnico PITRA-LanammeU$C R$, vol. 7, n.o 1, p. 5, 2016.
Ángel Gustavo Moyolema Chaglla: Licenciado en Ciencias de la Educación, Mención Físico Matemáticas. Magister en Matemática Aplicada.

Correo electrónico: angelgus@hotmail.es

Francisco Eduardo Toscano Guerrero: Ingeniero Civil, Especialista en Gestión y Desarrollo de Instituciones Educativas, Magister en Docencia Universitaria y Administración Educativa, Magister en Matemática Aplicada.

Correo electrónico: fe.toscano@uta.edu.ec

Byron Miguel Toalombo Rojas: Ingeniero Mecánico. Magister en Matemática Aplicada.

Correo electrónico: byronmtr@gmail.com

Recibido: 23 de noviembre de 2021

Aceptado: 28 de diciembre de 2021 